\title{
Electron Transport of a Linac Coherent Light Source (LCLS) Using the SLAC Linac
}

\section{Introduction}

A linac configuration prov ding a low emittance high peak current electron beam is u.rder study for a potential Linac Coherent Light Source (LCLS) based on the SLAC accelerator [1]. The parameters of the final electron bunch are nearing the technological limits of present accelerators in both transverse and longitudinal phase space. In this note we describe a layout of the RF gun, linac, and bunch compressors to deliver the required bunch properties.

We consider a bunch that is generated by an if gun and accelerated to $7 \mathrm{GeV}$ in $900 \mathrm{~m}$ of SLAC linac structure before it enters the wiggler. We assume that the rf gun generates a gaussian beam with an energy of $10 \mathrm{MeV}$, a population $N=6 \times 10^{9} \mathrm{e}^{-}$, an rms length $\sigma_{z}=0.5 \mathrm{~mm}$, an rms energy spread $\sigma_{\delta}=0.2 \%$, and normalized rms emittances $\gamma \epsilon_{x, y}=3 \mathrm{~mm}$-mrad. At the end of the linac, we require that the peak current $\hat{I} Z 2.5 \mathrm{kA}$ and the peak-toptik energy spread $\Delta \delta \$ 0.2 \%$.

To obtain the required high peak current, we need tu compress the bunch length by a factor greater than 10 . In deciding at what position in the linac to compress we need to consider three issues: (i) the longitudinal wakefield in the linac, $t^{\prime}, 1 s$...creases the beam's energy spread and is harder to compensate with short bunches, (ii) the transverse wakefield and if deflections in the linac, these increase the transverse emittance of the beam and are more severe for long bunches, and (iii) the effects of phase and current jitter winich will change the bunch length and therefore the peak current of the beam.

In the next sections, we will describe how we compress the bunch to meet these three criteria. Then, we will briefly describe the bunch compressor optics and finally we will mention some details specific to the SLAC site.

\section{Longitudinal and Transverse Wakefields}

Previously, we have studied the longitudinal and transverse wakefield effects in the linac [2]; the results are summarized in Fig. 1. Here, we have plotted the peak-topeak energy spread in the beam and the transverse emittance dilution versus the bunch length after acceleration from $100 \mathrm{MeV}$ to $7 \mathrm{GeV}$ in the linac. The transverse point: are calculated from the average of 10 random distributions of alignment errors with rms's typical of the SLAC linac. Finally, no emittance correction trajectory bumps have been utilized [3]; aithough the use of trajectory bumps could reduce the emittance dilution, we have chosen not to include them to select more conservative parameters.

* Work supported by Department of Energy, contract DEAC03-76SF00515.

$$
\text { MATER }
$$

Presented at the Particle Accelerator Conference (PAC 93), Washington, DC, May 17-20, 1993

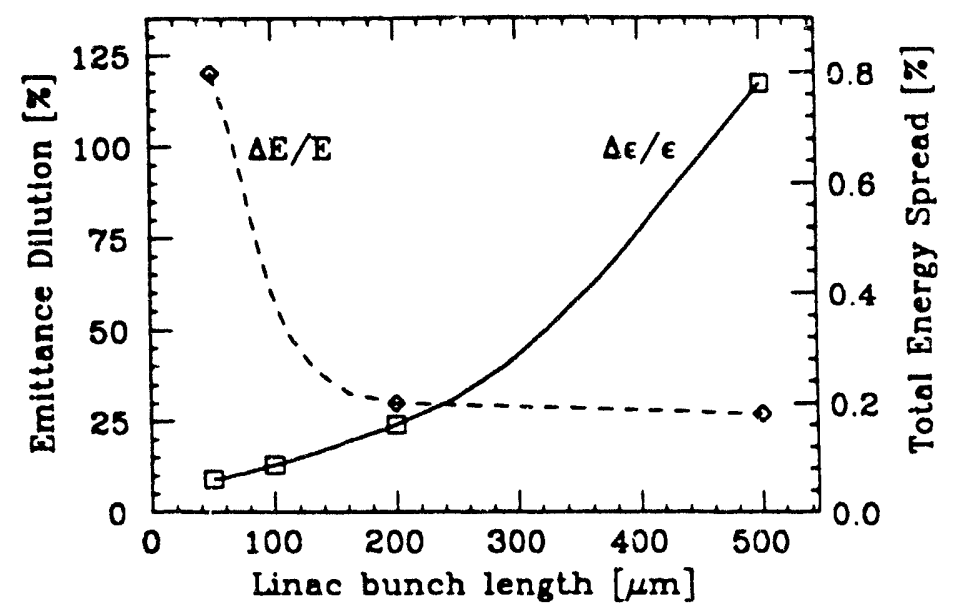

Fig. 1 Transverse emittance dilution (solid) and peak-to-peak energy spread (dashed) as a function of the linac bunch length. The emittance dilution is in units of the initial emittance $\gamma \varepsilon_{x, y}=3 \mathrm{~mm}$-mrad and the energy spread is in percent with a scale on the right. The plotting symbols are the values calculated.

As Fig. 1 shows, the transverse emittance dilution forces us to consider partially compressing the bunch before the main acceleration but the energy spread induced by the longitudinal wakefield prevents us from fully compressing right at the beginning. Thus, in Ref. 2 we concluded that we should compress to $\sigma_{z} \approx 200 \mu \mathrm{m}$ at roughly $100 \mathrm{MeV}$ and then fully compress at the end of the acceleration to achieve the necessary peak current.

\section{Jitter}

Previous work, described in Ref. 2 ignored the sensitivity of the peak current to phase and intensity jitter. The phase jitter arises from timing errors between the laser for the RF gun and the RF generated by the klystrons; the intensity jitter arises from the intensity jitter of the laser. Both of these effects will change the correlation between the energy deviation $(\delta)$ and longitudinal position $(z)$ along the bunch $\langle\delta z\rangle$. In the case of phase jitter, the $\langle\delta z\rangle$ correlation varies because of the non-linearity of the RF while with intensity jitter the variation occurs because a more intense bunch generates larger wakefields.

A simple single stage bunch compressor consists of an acceleration section, where an energy-longitudinal position correlation is introduced, followed by a dispersive region where the path length depends linearly upon the energy deviation, i.e. the $R_{56}$ transfer matrix element is non-zero; this rotates the bunch in longitudinal phase space. But, when the $\langle\delta z\rangle$ correlation is changed, the degree of rotation changes and thus phase and intensity jitter causes the compressed bunch length and peak current to change. 


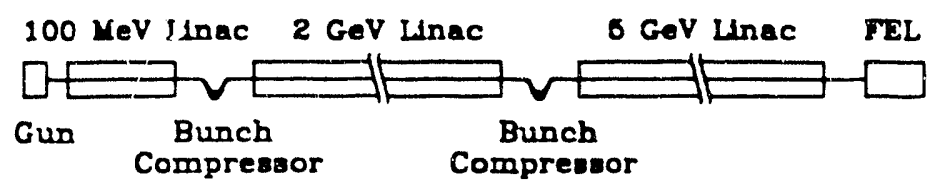

Fig. 2 Schematic illustration of beam transport.

In the LCLS, the longitudinal emittance from the RF gun is extremely small and thus we cannot operate with a fully compressed bunch; we must either under-compress or over-compress. Therefore, the longitudinal phase space is either rotated less than $90^{\circ}$ or it is rotated more than $90^{\circ}$. In this case, changes of the $\langle\delta z\rangle$ correlation cause a linear variation of the bunch length. For example, if we are under-compressing and the $\langle\delta z\rangle$ correlation decreases, the bunch length will increase. In contrast, if the correlation increases, the bunch length decreases. The opposite is true if we over-compress the bunch.

With phase jitter the bunch length change can be simply estimated. When the longitudinal emittance is extremely small, we can neglect the uncorrelated energy spread and calculate the bunch length after a single compression:

$$
\sigma_{z}(\Delta \phi)=\sigma_{20}\left|1-R_{56} k_{R F} \frac{\sin (\phi+\Delta \phi)}{\cos \phi}\right|,
$$

where $\Delta E=V_{R F} \cos \phi \gg E_{0}, \sigma_{z 0}$ is the initial bunch length, $\phi$ is the nominal RF phase and $\Delta \phi$ is the phase jitter. Now, the change in the bunch length is:

$$
\frac{1}{\sigma_{z}^{\star}} \frac{d \sigma_{z}}{d \Delta \phi}=\frac{\sigma_{z 0}^{\star}}{\sigma_{z}^{\star}}\left[\left(\frac{\sigma_{z}^{\star}}{\sigma_{z 0}^{\star}} \pm 1\right) \cot \phi\right],
$$

where the ${ }^{*}$ denotes the design values and the negative sign corresponds to under-compressing while the positive sign is for over-compressing. Notice that the sensitivity depends upon the compression factor $\sigma_{z} / \sigma_{20}$ and upon the $\mathrm{RF}$ phase, although the RF phase is also determined by the required energy gain and the correlated energy spread.

At this point, we can perform a similar calculation to include the effect of a second compression. In this case, we find two terms in the expression that can be chosen to cancel. Assuming that the bunch is forward of the RF crest so that the bunch head has a bigher energy than the tail, the two terms can cancel provided that the second compression is an over-compression; this can be understood from the simple arguments given above and noting that the phase jitter is under- or over-compressed along with the bunch length. If the bunch is behind the RF crest, then the second compression must be an under-compression for the two terms to cancel. Finally, similar calculations can be made for the intensity changes, but the results are poorer because the effects are more non-linear.

Thus, to satisfy all three conditions: preservation of the transverse emittance, reduction of the longitudinal energy spread, and reduction of the jitter sensitivity, we propose compressing in two stages. We would under- or over-compress once near the beginning of the linac and then over-compress in the middle of the acceleration; this achieves the desired high peak current while causing the jitter effects of the two compressors to cancel. The scheme is illustrated schematically in Fig. 2.

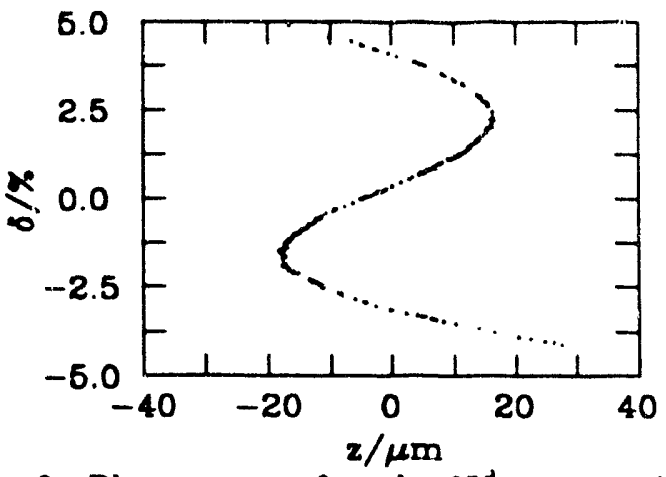

Fig. 3. Phase ace after the $2^{\text {nd }}$ compression. The bunch head points to negative values of $z$.

\section{Longitudinal Phase Space Simulations}

As described, without wakefields \& solution that has very relaxed jitter tolerances can be found analytically. The wakefields, however, make the problem more complicated. So we bave developed a computer program to study the development of longitudinal phase space, one that considers the effects of both the longitudinal wakefields in the SLAC linac [4] and the curvature of the of wave, and that does compression to first order. A parameter search was done to find good a solution. One example solution is sketched in Fig. 2. We first run the beam at $18^{\circ}$ with respect to the $\mathrm{of}$ crest to $112 \mathrm{MeV}$, where with an $R_{66}=2.3 \mathrm{~cm}$ we compress to an $\mathrm{rms}$ length of $0.25 \mathrm{~mm}$. (Our convention is that a negative phase is toward the front of the bunch. Therefore a positive $R_{56}$ will move a high energy particle more toward the back of the bunch.) Next we run the beam at $54^{\circ}$ up to $2.0 \mathrm{GeV}$, where with an $R_{56}=0.97 \mathrm{~cm}$, we over-compress to a double horned distribution with a full width of $35 \mu \mathrm{m}$ (phase space is shown in Fig. 3). Then finally we accelerate at the top of the rf crest to $7.0 \mathrm{GeV}$. In the simulations, we have used an acceleration gradient of $14 \mathrm{MeV} / \mathrm{m}$ which is $25 \%$ less than that achieved in the SLAC linac. This will allow for flexibility in the operation of the LCLS.

The final beam phase space, the bunch shape, and the energy distribution are shown in Fig. 4. Note that between the horns of the bunch distribution the peak current is everywhere $\geq 5.0 \mathrm{kA}$ and that the full-width of the energy distribution is $0.04 \%$, both of which satisfy our requirements. Let us define the jitter tolerance as the amount of change in a parameter that changes the full-width of the bunch distribution by $10 \%$. We find that in this example the tolerance to incoming current jitter is $\pm 2.2 \%$ and to incoming phase jitter is $\pm 0.45^{\circ}$. For comparison, when we consider a scheme that uses only one compression, at $100 \mathrm{MeV}$, to obtain the desired peak current we find an unacceptable energy distribution width of $1.4 \%$, a current jitter tolerance of $\pm 2 \%$, and a phase jitter tclerance of $\pm 0.15^{\circ}$. Alternately, if we consider a single compression at the end of the acceleration, we can achieve the desired energy bandwidth, but the phase jitter tolerance is reduced to $0.04^{\circ}$ and the transverse emittance growth is too large.

The branches in phase space that point upward and downward in Fig. 4c will not contribute to lasing, and it might be desirable to remove them. This can be conve- 


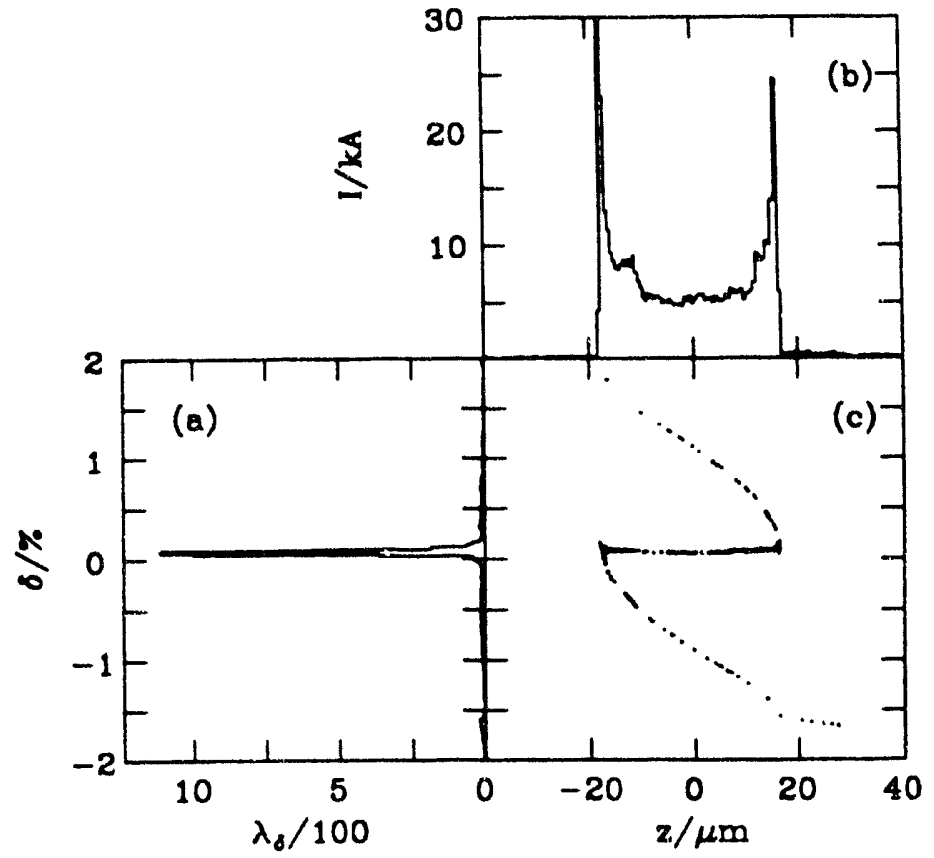

Fig. 4. The energy spectrum (a), the charge distribution (b), and the shape of phase space (c) at the end of the linac.

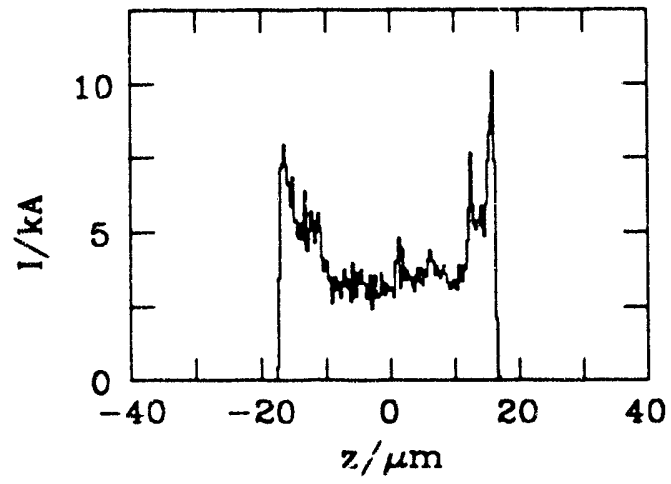

Fig. 5. The bunch shape for the example of Fig. 4 except that after the $2^{\text {nd }}$ compression particles with energy deviations $\delta>2.0 \%$ and $\delta<-1.5 \%$ are removed.

niently done immediately after the second compression by clipping in energ". By removing particles with relative energy variation $\delta>2.0 \%$ and $\delta<-1.5 \%$, which will remove about half of the beam, we obtain the bunch distribution shown in Fig. 5 . Now $I \geq 3.0 \mathrm{kA}$ between the horns.

One important comment about these results: The wakefields obtained from Ref. 4 were not intended to be used for bunches as short $35 \mu \mathrm{m}$. We estimate that the wakefield effect after the second compressor may be underestimated by as much as $25 \%$. If we artificially increase the wakefield by $25 \%$ in the last acceleration section we find that the final bandwidth becomes $0.2 \%$, which can be reduced by changing the compressor parameters. Note that with the very short bunches that we have after the second compressor we can't compensate the correlated energy spread by going off crest in the last acceleration region; instead, we compensate by adjusting the correlated energy spread at the exit of the second compressor which is shown in Fig. 3. Finally, we intend to obtain a better wakefield for future work.

Solutions have also been found which do not have a double horned bunch distribution, but rather are more gaussian-like, though more highly peaked and with longer tails. Some of these solutions have much looser jitter tolerances than presented here, however they tend to have larger energy bandwidths than desired. Note that for the example presented here, after the second compression the bunch tail is at higher energy than the head, which will therefore tend to compensate the effect of the longitudinal wakefield in the final acceleration section.

\section{Compressor Optics}

The optics of the bunch compressors are quite straightforward. We have studied various optical solutions for the LCLS; a similar study is described in Ref. 5. The emittance dilution due to the bunch compressors should be small because the required $R_{36}$ 's are small and the beam energy is high so that space charge is not significant.

\section{SLAC Site Specifics}

The upgrade of the SLAC linac to include an RF gun, preaccelerator, and two bunch compressors is straightforward. The bulk of the acceleration would be done in the main SLAC linac with existing equipment. The first and second compressors would be either magnetic s-bends or chicanes. The injection-extraction locations would be provided for by removing $12 \mathrm{~m}$ accelerating sections. The gun would likely be placed about $700 \mathrm{~m}$ from the end of the linac to provide sufficient acceleration downstream with (1) $8 \mathrm{GeV}$ maximum beam energy, (2) $10 \%$ of the klystron:; as spare or in maintenance, (3) sufficient energy feedbac!. overhead, (4) bunch phase offsets up to 55 degrees, and (5) space for length compressors. Specific plans are under investigation. Finally, it should be noted that, operationally, single bunches of $3 \times 10^{10}$ particles with vertical emittances of $3 \mathrm{~mm}$-mrad have been stably transported in the SLAC linac [6].

\section{References}

[1] H. Winick, et. al., “A 2-4nm Linac Coherent Light Source (LCLS) Using the SLAC Linac," Presented to this conference, Washington, DC. (1993).

[2] C. Pellegrini, et. al., "A 2 to $4 \mathrm{~nm}$ High Power FEL on the SLAC Linac," Presented at the 19th Int. FEL Conf., Kobe, Japan (1992).

[3] J. T. Seeman, F.-J. Decker, I. Hsu, "The Introduction of Trajectory Oscillations to Reduce Emittance Growth in the SLC Linac," Proc. of the $15^{\text {th }}$ Int. Conf. on High Energy Acc., Hamburg, Germany (1992).

[4] K. Bane and P.B. Wilson, Proc. of the $11^{\text {th }}$ Int. Conf. on High-Energy Acc., CERN (1980), p.592.

[5] J. T. Seeman and R. Holtzapple, "Short Bunch Length Test Area Using the SLAC Linac," Presented to this conference, Washington, DC. (1993).

[6] C. Adolphsen, et. al., "Flat Beam Studies in the SLC, Linac," Presented to the conference, Washington, DC. (1993). 
I

$\checkmark$
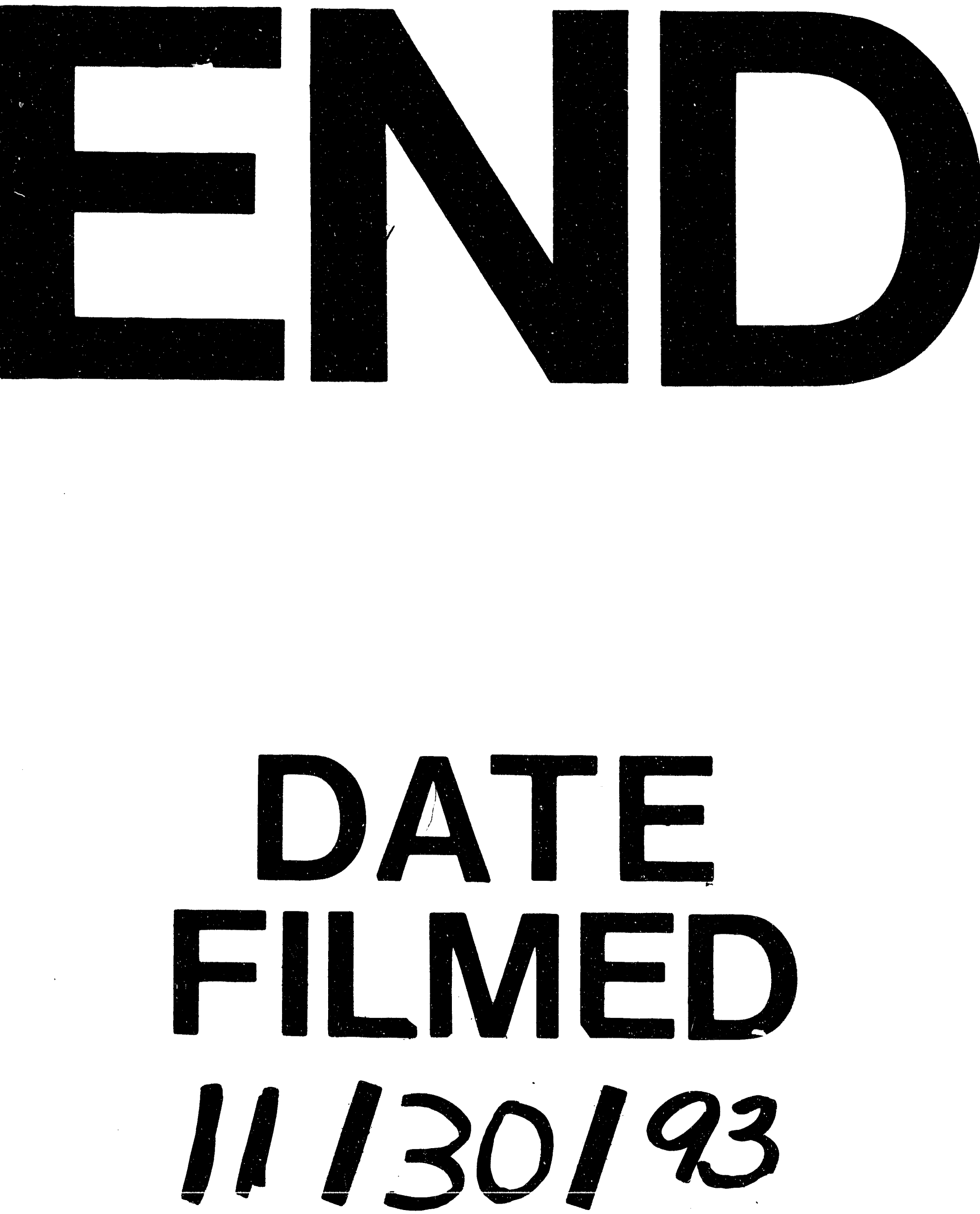
